\title{
3D printed microfluidic chips: Futuristic approach for rapid, affordable, point-of-care diagnostics and biomedical research
}

Point-of-care (POC) or bedside diagnostics are becoming the ultimate need for improving medical treatment and better healthcare. The advantages of this strategy are rapid diagnosis, affordable cost, requirement of less expertise and can be performed in resource limited healthcare settings. The development of POC diagnostics requires an inter-disciplinary approach which can improve the diagnostic parameters like sensitivity and specificity and also to enhance analysis with low sample volume. Advancements in microfluidic technology enabled the researchers to develop such diagnostics, integrated with sensors for rapid and easy detection.

Microfluidics, as the name implies, deals with micro quantities of sample volume, which can be controlled for better results. Apart from diagnostics, it has been implemented in several research oriented applications like animal cell growth, tissue engineering, modelling human organs, etc. Recently, scientists from University of Illinois, developed a microfluidic chip that can diagnose sepsis in less than 30 minutes with high accuracy, by separating and counting the white blood cells and neutrophils that express CD64, with a sample volume of 10 microlitres. $^{1}$

In India, several premier institutes like Indian Institute of Technology, Mumbai (IIT-B); Centre for Cellular and Molecular Biology (CCMB), Hyderabad; National Centre for Biological Sciences (NCBS), Bangalore; Indian Institute of Science (IISc), Bangalore, are involved in microfluidic research and product development for different applications. For example, researchers from IISc are working on micro and nano fluidics for DNA sequencing, biosensing, probing cells in microfluidic device, etc. Optics and Microfluidics Instrumentation lab, IISc, has developed several microfluidic chips for microscopy, malaria diagnosis, etc. The Microfluidics and Biological physics laboratory at IIT-B is working on developing chips for cell sorting, detection of sickle cell disease and studying cell interactions and population dynamics of bacteria. India is advancing in microfluidic research for single-step cell lysis and nucleic acid extraction from waterborne-pathogens, assessing chemotactic behavior of spermatozoa, enhanced cryopreservation of human mesenchymal stem cells. ${ }^{2-4}$

The crucial step in developing such chips is microfabrication, which decides the cost and efficiency of each model. The material and technology used for microfabrication is particularly important. Traditional microfluidic chip fabrication methods such as soft lithography and polydimethylsiloxane (PDMS), needs expertise and expensive equipment. The introduction of $3 \mathrm{D}$ printers in microfluidic research made the fabrication process faster and reduced the manufacturing cost, significantly. Microfluidic chips can be fabricated through various 3D printing strategies such as Stereolithography (SLA), Fused Deposition Modeling (FDM), Selective Laser melting and Sintering (SLS) and Multi-Jet Modeling (MJM). ${ }^{5}$ The 3D printing based microfluidic chip development training and services are being provided by various national and private laboratories like Achira Labs, Bangalore; In Stem, Bangalore.

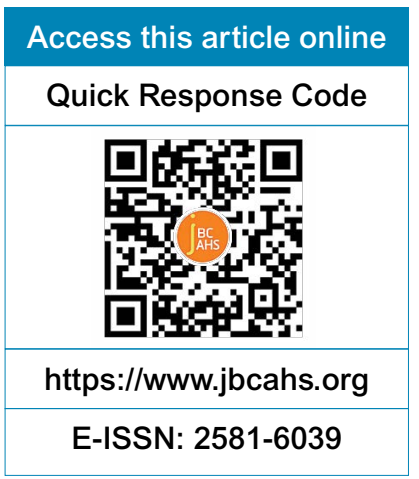

The affordable cost of this technology is making it so popular, compared to expensive high throughput technologies. For example, the cost of genetic testing through currently available technologies like TaqMan assays, Next Generation sequencing, are too high, which is not affordable for a patient in low economic status. Whereas, 3D printed microfluidic chips can provide comparable results in surprisingly low cost through integration of nucleic acid extraction, amplification and detection in a single chip.

How to Cite: Benet Bosco Dhas D, Adithan C. Editorial, 3D printed microfluidic chips: Futuristic approach for rapid, affordable, point-of-care diagnostics and biomedical research. J Basic Clin Appl Health Sci. 2019;2:1-2 
Indian scientists working in health care institutions need to focus their research in developing 3D printed microfluidic chips based diagnostic kits for providing affordable and rapid diagnostic services in infectious diseases, pharmacogenetic testing and several other medical applications.

For example, Chowdhury et al., developed a microfluidic chip based polymerase chain reaction (PCR) and electrophoresis platform for genotyping single nucleotide polymorphisms (SNPs) in thiopurine S-methyl transferase gene to assess the adverse effects associated with thiopurine therapy. ${ }^{6}$ It is also possible to develop integrated microfluidic chips that can perform nucleic acid extraction, PCR amplification and genetic analysis in a single chip. ${ }^{7}$

The utilization of loop mediated isothermal amplification methods (LAMP assay) gives the benefits of reduced time for analysis, easy detection and decreased overall-cost of the chip. Such integrated chips does not require any expensive equipment, rather than a water bath / dry bath / handwarmer, which can provide a temperature of $60-65^{\circ} \mathrm{C}$. This comprehensive inter-disciplinary approach of developing 3D printed microfluidic chip with LAMP assay for genotyping will help in rapid and affordable bedside diagnosis of genotype-guided drug dosage.

\section{Benet Bosco Dhas D', Adithan C²}

${ }^{1}$ Department of Biotechnology, Vignan's Foundation for Science, Technology and Research, Vadlamudi, Guntur, Andhra Pradesh - 522213. Email: benetbiotech@gmail.com

${ }^{2}$ Sri Balaji Vidyapeeth Pillaiyarkuppam Pondicherry - 607402 Email: adithan50@gmail.com

\section{References}

1. Zhang Y, Zhou Y, Li W, Lyons V, Johnson A, Venable A, et al. Multiparameter affinity microchip for early sepsis diagnosis based on CD64 and CD69 expression and cell capture. Anal Chem. 2018;90:7204-11.

2. Kamat V, Pandey S, Paknikar K, Bodas D. A facile one-step method for cell lysis and DNA extraction of waterborne pathogens using a microchip. Biosens Bioelectron. 2018;99:62-9.

3. Bhagwat S, Sontakke S, K D, Parte P, Jadhav S. Chemotactic behavior of spermatozoa captured using a microfluidic chip. Biomicrofluidics. 2018;12(2):024112.

4. Bissoyi A, Bit A, Singh BK, Singh AK, Patra PK. Enhanced cryopreservation of MSCs in microfluidic bioreactor by regulated shear flow. Sci Rep. 2016;6:35416.

5. Zhou Y. The recent development and applications of fluidic channels by 3D printing. J Biomed Sci. 2017;24:80.

6. Chowdhury J, Kagiala GV, Pushpakom S, Lauzon J, Makin A, Atrazhev A, et al. Microfluidic platform for single nucleotide polymorphism genotyping of the thiopurine S-methyltransferase gene to evaluate risk for adverse drug events. J Mol Diagn. 2007;9:521-9.

7. Wang H, Zhang X, Xu X, Zhang Q, Wang H, Li D, et al. A portable microfluidic platform for rapid molecular diagnostic testing of patients with myeloproliferative neoplasms. Sci Rep. 2017;7:8596. 